\title{
Observations From a Mouse Model of Forebrain Voa1 Knockout: Focus on Hippocampal Structure and Function
}

\author{
Ke Ma ${ }^{1,2}$, Na-Ryum Bin ${ }^{2}$, Shan Shi ${ }^{1,2 *}$, Hidekiyo Harada ${ }^{2}$, Yoh Wada ${ }^{3}$, \\ Ge-Hong-Sun Wada ${ }^{4}$, Philippe P. Monnier ${ }^{2,5,6}$, Shuzo Sugita ${ }^{2,6 *}$ and Liang Zhang ${ }^{2,7}$ \\ ${ }^{1}$ Department of Pediatric Outpatient, The First Hospital of Jilin University, Jilin, China, ${ }^{2}$ Krembil Research Institute, University \\ Health Network, Toronto, ON, Canada, ${ }^{3}$ Division of Biological Science, Institute of Scientific and Industrial Research, Osaka \\ University, Osaka, Japan, ${ }^{4}$ Department of Biochemistry, Faculty of Pharmaceutical Sciences, Doshisha Women's College, \\ Kyoto, Japan, ${ }^{5}$ Department of Ophthalmology, University of Toronto, Toronto, ON, Canada, ${ }^{6}$ Department of Physiology, \\ University of Toronto, Toronto, ON, Canada, 'Department of Medicine, University of Toronto, Toronto, ON, Canada
}

OPEN ACCESS

Edited by:

Jerzy W. Mozrzymas,

Wroclaw Medical University, Poland

Reviewed by:

Pavel Ivanovich Ortinski, University of Kentucky, United States

Zhe Jin,

Uppsala University, Sweden

*Correspondence:

Shan Shi

norri961094@gmail.com

Shuzo Sugita

shuzo.sugita@uhnresearch.ca

Received: 22 July 2019 Accepted: 11 October 2019 Published: 21 November 2019

Citation:

Ma K, Bin N-R, Shi S, Harada H, Wada Y, Wada G-H-S, Monnier PP, Sugita $S$ and Zhang $L$ (2019) Observations From a Mouse Model of Forebrain Voa1 Knockout:

Focus on Hippocampal Structure and Function.

Front. Cell. Neurosci. 13:484. doi: 10.3389/fncel.2019.00484
Voa protein is a subunit of V-ATPase proton pump which is essential to acidify intracellular organelles including synaptic vesicles. Voa1 is one of the four isoforms of Voa family with strong expression in neurons. Our present study was aimed to examine the role of Voa1 protein in mammalian brain neurons. To circumvent embryonic lethality, we generated conditional Voa1 knockout mice in which Voa1 was selectively deleted from forebrain pyramidal neurons. We performed experiments in the Voa1 knockout mice of ages 5-6 months to assess the persistent effects of Voa1 deletion. We found that the Voa1 knockout mice exhibited poor performance in the Morris water maze test compared to control mice. In addition, synaptic field potentials of the hippocampal CA1 region were greatly diminished in the Voa1 knockout mice when examined in brain slices in vitro. Furthermore, brain histological experiments showed severe degeneration of dorsal hippocampal CA1 neurons while CA3 neurons were largely preserved. The CA1 neurodegeneration was associated with general brain atrophy as overall hemispheric areas were reduced in the Voa1 CKO mice. Despite the CA1 degeneration and dysfunction, electroencephalographic recordings from the hippocampal CA3 area revealed aberrant spikes and non-convulsive discharges in the Voa1 knockout mice but not in control mice. These hippocampal spikes were suppressed by single intraperitoneal injection of diazepam which is a benzodiazepine $\mathrm{GABA}_{A}$ receptor enhancer. Together these results suggest that Voa1 related activities are essential for the survival of the targeted neurons in the dorsal hippocampal CA1 as well as other forebrain areas. We postulate that the Voa1 knockout mice may serve as a valuable model for further investigation of V-ATPase dysfunction related neuronal degeneration and functional abnormalities in forebrain areas particularly the hippocampus.

Keywords: V-ATPase, spatial memory, glutamate transamination, neuronal degeneration, synapse, brain slice, EEG, conditional knock out mice 


\section{INTRODUCTION}

Vacuolar $\mathrm{H}^{+}$-ATPases or V-ATPases are multi-subunit proton pumps that acidify a wide array of intracellular organelles including synaptic vesicles (Moriyama and Futai, 1990) and are crucial for many fundamental processes including endocytosis, signaling molecule processing, protein sorting, trafficking, lysosomal as well as autophagosomal enzyme activation (Forgac, 2007; Cotter et al., 2015). In general, V-ATPases consist of a Vo proton-translocation domain and a V1 pump domain and two associated proteins Ac45 and ATP6AP2. The largest $(\sim 110 \mathrm{kDa})$ subunit of the Vo domain, Voa, is an integral membrane protein with four isoform genes (a1-a4) identified in human, mouse and Drosophila. While Voa1, Voa2, and Voa3 are widely expressed, Voa1 is more strongly expressed in the brain, whereas Voa3 is primarily expressed in osteoclasts (Perin et al., 1991; Nishi and Forgac, 2000; Toyomura et al., 2000).

The proteolipid pore-forming Vo domain of V-ATPases is thought to be required for membrane fusion downstream of Soluble N-ethylmaleimide-sensitive factor attachment protein receptor (SNARE) complex (Peters et al., 2001; Hiesinger et al., 2005; Peri and Nüsslein-Volhard, 2008; Saw et al., 2011; Wang et al., 2014). For example, loss-of-function mutations in a neuron-specific Voa subunit (Vha100-1) exhibit defects in a late stage of synaptic exocytosis, which suggests that Vha100-1 is a regulator of synaptic vesicle fusion efficiency downstream of SNARE-dependent vesicle priming in Drosophila (Hiesinger et al., 2005). In mouse hippocampal neurons, Voal-containing V-ATPase does not play a direct role in synaptic vesicles fusion but modulates neurotransmitter release upstream of docking by facilitating fusion of fully acidified and neurotransmitterloaded synaptic vesicles (Bodzeta et al., 2017). While Voal over-expression does not produce significant alterations in glutamatergic synaptic transmission in rat hippocampal neurons (Chanaday and Kavalali, 2018), Voal dysfunction is implicated in neurological diseases and relevant models (Colacurcio and Nixon, 2016). In particular, impaired glycosylation and instability of Voa1 leading to deficiencies in lysosomal V-ATPase assembly are recognized in a mouse model of Alzheimer Disease (Lee et al., 2015). Misrouted V-ATPase Voal is a major cause of dysregulated lysosomal acidification in neurons of a mouse model of severe infantile neuronal ceroid lipofuscinoses (Bagh et al., 2017). Together these findings call further investigation into the role of Voal protein in mammalian brain neurons. Our present study was aimed to provide more information in this area with a particular focus on the hippocampus.

Global knockout of this gene was known to be embryonic lethal (Dickinson et al., 2016). Therefore, we generated a conditional Voal knockout (Voal cKO) model using CaMK2-Cre mice (Tsien et al., 1996) to selectively delete Voa1 in pyramidal neurons of forebrain areas including the hippocampus. By examining Voal cKO mice of ages 5-6 months, we tested the hypothesis that chronic Voal deletion may cause pronounced structural and functional abnormalities in targeted hippocampal neurons.

\section{MATERIALS AND METHODS}

\section{Animals}

All mice were maintained in a vivarium on a 12-h light on/off cycle and at a temperature between 22 and $23^{\circ} \mathrm{C}$. Mice were housed in standard cages with food and water ad libitum. All experiments performed were reviewed and approved by the animal care committee of the University Health Network in accordance with the Canadian Guidelines for Animal Care.

Voa1 flox mice (Atp6v0a1_tm1c_H11) were obtained from the Center for Phenogenomics in Toronto. Diagram of the floxed allele in comparison with WT allele is shown in Supplementary Figure S1A. The genetic background of ES cells to generate the Voal flox mice is C57BL/6N. We obtained C57BL/6 mice with CaMKIIalpha-Cre (Tsien et al., 1996) from the Jackson Laboratory (\#005359; B6.Cg-Tg(CaMKIIalpha-cre)T29-1Stl/J) to generate conditional knockout mice. Genotyping of each animal was performed using PCR by extracting genomic DNA from the tail. Specifically, mouse tails were collected and genomic DNA was obtained by an alkaline lysing method where the samples were lysed with $50 \mathrm{mM} \mathrm{NaOH}$ at $95^{\circ} \mathrm{C}$ for $2 \mathrm{~h}$. Then $0.5 \mathrm{M}$ Tris- $\mathrm{HCl}(\mathrm{pH} 8.0)$ was added to neutralize $\mathrm{pH}$. The supernatant was directly used in PCR for genotyping. To detect the difference between wild type vs. floxed allele, PCR was performed using a forward primer $5^{\prime}$-ACCTGGTGTATTCCATTCACTC $3^{\prime}$ and a reverse primer $5^{\prime}$-TTCTTCTGCCCCAGGATGATG$3^{\prime}$, which are located on the upstream and downstream of $5^{\prime}$ loxP site of exon 3 (see the diagram in Supplementary Figure S1A). For Cre recombinase PCR, a forward primer of 5'-GAACCTGATGGACATGTTCAGG-3' and a reverse primer of 5'-AGTGCGTTCGAACGCTAGAGCCTGT-3' were used. For all experiments, Voal flox mice without CaMKIIalpha-Cre were used as controls.

\section{Voa1 Knockout in Cultured Hippocampal Neurons}

Hippocampi were dissected out from postnatal day 0-1 Voa1 floxed mice of both sexes. Neurons were prepared and cultured as described previously (Kavalali et al., 1999) with minor modifications. Briefly, neurons were dissociated by treatment with trypsin $(10 \mathrm{mg} / \mathrm{ml})$ and DNase $(0.25 \mathrm{mg} / \mathrm{ml})$ for $10 \mathrm{~min}$ at $37^{\circ} \mathrm{C}$, triturated with a $200 \mu \mathrm{l}$ pipette tip and then plated onto $12 \mathrm{~mm}$ coverslips coated with Matrigel (BD Biosciences) and poly-L-ornithine. A plating density of two hippocampi per six coverslips was used. Culture media consisted of minimal essential media, $5 \mathrm{mg} / \mathrm{ml}$ glucose, $0.1 \mathrm{mg} / \mathrm{ml}$ transferrin, $0.5 \mathrm{mM}$ GlutaMAX, 5\% iron supplemented calf serum (Hyclone), 2\% B-27 supplement, $1 \mu \mathrm{M}$ cytosine arabinoside, 50 units $/ \mathrm{ml}$ of penicillin, and $50 \mu \mathrm{g} / \mathrm{ml}$ of streptomycin. Cultures were maintained at $37^{\circ} \mathrm{C}$ in a humidified incubator gassed with $95 \%$ air and $5 \% \mathrm{CO}_{2}$. Cells were infected with lentiviral particles that co-expressed GFP and blasticidin S resistance gene (bsr, control) or GFP and Cre recombinase gene (KO) on day in vitro 1 (DIV 1). Whereas control neurons easily survived more than DIV 21, KO neurons started showing signs of deterioration on DIV 13 and completely degenerated on DIV 15. Therefore, both control and KO neurons were harvested on 
DIV 13 with a cell scraper and subjected to Western blot analysis in which each lane of SDS-PAGE was loaded with $5 \mu \mathrm{g}$ homogenates.

\section{Voa1 Expression in Wild Type Mice}

Immunofluorescence microscopy in brain sections was used to examine Voal expression. The mouse was anesthetized by sodium pentobarbital $(70 \mathrm{mg} / \mathrm{Kg}$, intra-peritoneal injection) and transcardially infused with PBS and then with 4\%PFA. The brain was removed and further fixed in 4\%PFA for $>24 \mathrm{~h}$ and then with a hypertonic (with $30 \%$ sucrose) formalin solution for $>24 \mathrm{~h}$. Coronal sections of $30 \mu \mathrm{m}$ thickness were obtained and then incubated with blocking buffer (PBS containing $0.1 \%$ Triton X-100 and 2\% BSA) for $45 \mathrm{~min}$. Voal antibody (IgY, chicken monoclonal antibody generated from Dr. Yoh Wada lab of Osaka University, Japan; see also Sun-Wada et al., 2011) was added to the blocking buffer at 1:100 dilution. The sections were incubated with the antibody at $4^{\circ} \mathrm{C}$ overnight. Then, they were washed with PBST (PBS containing 0.1\% of Tween 20) three times and incubated with Alexa Fluor goat anti-chick antibody $(1: 1,000)$ in dark for $1 \mathrm{~h}$. Brain sections similarly stained by the goat anti-chick antibody alone (without the chicken monoclonal Voal antibody) were used to control non-specific signals. For subsequent DAPI staining, the samples were washed with PBS three times before and after 5 min-incubation with $0.1 \mathrm{mg} / \mathrm{ml}$ DAPI (product\# ab228549; abcam.com). The stained samples were mounted on the glass slide with fluoromount $\mathrm{G}$ (Southern Biotech, Birmingham, AL, USA) and covered by a cover slip. Images were obtained using a fluorescent microscope (Olympus BX61) and Olympus DP70 digital camera.

\section{Morris Water Maze Test}

We used a maze protocol with 9-day trials and two-probe tests (Bin et al., 2018). Mice underwent visible platform trials for 3 days and then hidden platform trials for 6 days $(90 \mathrm{~s}$ per trial and four trials per day). The inter-trial intervals were roughly $10-15 \mathrm{~min}$. For visible platform trials, if the mice were unable to find the platform within $90 \mathrm{~s}$, they were guided to the platform by the experimenter's hand. For hidden platform trials, the procedure was identical to visible platform trials with the exception that the platform was submerged underwater at a depth of $1.5 \mathrm{~cm}$ and the platform location was changed. The distances and latencies individual mice needed to find the platform during visible and hidden platform trials were measured. For the probe tests, the times the mice spent in searching the pool quadrant where the platform was previously located were measured. We used Micro-Manager 2.0 (Open Source Microscopy Software) for recording and Image (National Institute of Health, Bethesda, MD, USA) software for analysis in the Morris water maze test.

\section{Electrophysiological Recordings in Brain Slices}

Brain slice preparation and in vitro recordings were done as previously described (Bin et al., 2018; Song et al., 2018a). Briefly, the mouse was anesthetized by sodium pentobarbital
(70 $\mathrm{mg} / \mathrm{Kg}$, intra-peritoneal injection) and transcardially infused with cold, dissection-only artificial cerebrospinal fluid (ACSF) before brain dissection. Brain slices of $0.4 \mathrm{~mm}$ thickness were obtained using a vibratome (Leica VT1200) in ice-cold dissection-only ACSF. Slices encompassing dorsal hippocampal areas (roughly corresponding to Bregma -1.46 to $-2.18 \mathrm{~mm}$ ) were obtained via coronal sections. Slices encompassing ventral hippocampal areas were obtained via horizontal sections from the basal brain as the ventral hippocampus can be obtained at the transverse plane in these slices. The first three horizontal slices with clearly recognizable ventral hippocampal areas were collected for recordings. After vibratome section, slices were maintained in oxygenated $\left(5 \% \mathrm{CO}_{2}-95 \% \mathrm{O}_{2}\right)$ standard ACSF at room temperature $\left(21-22^{\circ} \mathrm{C}\right)$ for $1-6 \mathrm{~h}$ before recordings. The dissection-only ACSF contained (in $\mathrm{mM}$ ): sucrose 300, $\mathrm{KCl} 3.5, \mathrm{CaCl}_{2}$ 0.5, $\mathrm{MgCl}_{2}$ 6, HEPES 5 and glucose 20 ( $\mathrm{pH}$ adjusted to 7.4). The standard ACSF contained (mM): $\mathrm{NaCl} 125, \mathrm{KCl} 3.5, \mathrm{NaH}_{2} \mathrm{PO}_{4} 1.25, \mathrm{CaCl}_{2} 2, \mathrm{MgSO}_{4} 1.3$, $\mathrm{NaHCO}_{3} 25$ and glucose $10(\mathrm{pH}$ 7.35-7.4 when aerated with $5 \% \mathrm{CO}_{2}-95 \% \mathrm{O}_{2}$ ).

All recordings were done in a submerged chamber where each slice was perfused with oxygenated $\left(5 \% \mathrm{CO}_{2}-95 \% \mathrm{O}_{2}\right)$ standard ACSF at room temperature. Extracellular recording electrodes were made of thin-wall glass tubes (World Precision Instruments, Sarasota, FL, USA) and filled with a solution with $150 \mathrm{mM} \mathrm{NaCl}$ and $2 \mathrm{mM}$ HEPES ( $\mathrm{pH} 7.4$; resistance of 1-2 M $\Omega$ ). Local field potentials were recorded using an Axoclamp 2B amplifier with input frequencies set between $0-1,000 \mathrm{~Hz}$. Signals were amplified by 1,000 times and then digitized at $10,000 \mathrm{~Hz}$ (Digidata 1440 , Molecular Devices/Axon Instruments, Sunnyvale, CA, USA). A bipolar electrode, made of polyimide-insulated stainless steel wires (outer diameter $0.1 \mathrm{~mm}$; Plastics One, Ranoake, VA, USA), was placed in the CA2 striatum radium area to stimulate the Schaffer collateral-CA1 pathway. Constant current pulses $(0.1 \mathrm{~ms}$ duration, intensities of $10-150 \mu \mathrm{A})$ were generated by a Grass stimulator and delivered through a photoelectric isolation unit every 20 s (S88, Natus Neurology Incorporated-Grass Products, Warwick, RI, USA). Data acquisition, storage and analysis were completed using PClamp software (version 10, Molecular Devices).

Synaptic field potentials were evoked by paired stimuli (interval $25 \mathrm{~ms}$ ) with incrementing intensities of $10-150 \mu \mathrm{A}$ (10 $\mu \mathrm{A}$ per step) and recorded from the CA1 striatum radium area. Four consecutive responses evoked by a given intensity were averaged for measurements of presynaptic volley and synaptic potential amplitudes.

\section{Brain Histology}

Brain histological assessments were performed as previously described (Bin et al., 2018; Song et al., 2018a). Briefly, the mouse was anesthetized by sodium pentobarbital and transcardially infused with the dissection-only ACSF and then with $10 \%$ neutral buffered formalin solution. The brain was removed and further fixed in a hypertonic (with 20\% sucrose) formalin solution for $\geq 24 \mathrm{~h}$. Cryostat coronal sections of $25 \mu \mathrm{m}$ thickness were obtained and stained. Images were obtained using a slide scanner (Aperio digital pathology slide scanner AT2, 
Leica) at $20 \times$ magnification and analyzed using ImageScope (Leica) and ImageJ software. Hemispheric area, hippocampal area including CA1 and CA3 cell body layers were measured from sections corresponding Bregma $-1.7 \mathrm{~mm}$ to $-1.94 \mathrm{~mm}$ and Bregma $-3.16 \mathrm{~mm}$ to $-3.4 \mathrm{~mm}$. The densities of CA1 and CA3 cell body layers were measured from middle CA1 and CA3 regions (200 $\mu \mathrm{m}$ in length). Brightness and contrast of grayscale images were adjusted to distinguish cell body layers from adjacent dendritic layers. The areas (pixel numbers) of cell body layers were measured from adjacent three sections for each mouse.

\section{Electroencephalographic (EEG) Recordings}

Electrode implantation and electroencephalographic (EEG) recordings were performed as previously described (Bin et al., 2017; Song et al., 2018b). All electrodes were made with polyimide-insulated stainless-steel wires (outside diameter $0.1 \mathrm{~mm}$, Plastics One). Two pairs of bipolar electrodes were implanted bilaterally in middle-ventral hippocampal CA3 areas (Bregma $-2.9 \mathrm{~mm}$, lateral $2.5 \mathrm{mM}$ and depth $3.0 \mathrm{~mm}$ : Franklin and Paxinos, 1997). A reference electrode was positioned into a frontal area (Bregma $+2.0 \mathrm{~mm}$, lateral $1.0 \mathrm{~mm}$ and depth $0.5 \mathrm{~mm}$ ). We implanted electrodes in the middle-ventral hippocampal CA3 area because dorsal hippocampal areas were decreased in the Voal cKO mice and it was difficult to target the dorsal hippocampal CA3 in these mice. Implanted mice were allowed to recover for $\geq 1$ week prior to baseline monitoring. The locations of implanted electrodes were verified by later histological assessments if suitable.

EEG recordings were performed in free-moving mice. EEG signals were collected using a dual-channel AC microelectrode amplifier (model 1800, AM Systems, Sequim, WA, USA) with input frequencies set between 0.1 and $1,000 \mathrm{~Hz}$ and amplification by 1,000 times. Data digitization (at $5 \mathrm{KHz}$ ), acquisition, storage and analysis were done using the Molecular Devices system as described above. EEG spikes were recognized by intermittent events with large peak amplitudes ( $\geq 6$ times of standard deviation of background signals), simple or complex spike waveforms, and durations of 30-250 ms (El-Hayek et al., 2013). Spike incidences were measured from 50 to $70 \mathrm{~min}$ data segments collected during stable immobility or sleep, as hippocampal spikes manifested in these "inactive" behavioral states and EEG signals were minimally interfered by movement-related artifacts (El-Hayek et al., 2013). An event detection function (threshold search method) of PClamp software was used to detect spikes automatically, and detected events were then visually inspected and false events were rejected.

Diazepam was obtained in a clinically injectable form (Sandoz Canada Inc., Boucherville, QC, Canada) and diluted with saline for intraperitoneal injection ( $1.5 \mathrm{mg} / \mathrm{kg} /$ injection, El-Hayek et al., 2013). Saline injections were used as controls. Data collected within $8 \mathrm{~h}$ post diazepam or saline injections were compared in individual mice.

\section{Statistical Analysis}

All statistics were performed using Origin 9 (OriginLab, Northampton, MA, USA) or SPSS (IBM Corporation, Armonk, NY, USA). Data were presented as mean and standard error of mean (SEM) throughout the text and figures. Statistical significance was set at $p<0.05$. A student's $t$-test (independent or paired) was used for two group comparisons. A mixed repeated ANOVA was used for comparison between groups. A one-way repeated ANOVA was used for comparison within group.

\section{RESULTS}

Voal is widely expressed in the brain of wild type mice including the hippocampus as revealed by immunofluorescent imaging with Voa1 antibody (Supplementary Figure S2). The Voa1 cKO model we generated using CaMKII $\alpha$-Cre mice (Tsien et al., 1996) targets selectively forebrain pyramidal neurons particularly those in the dorsal hippocampal CA1. We, therefore, focused on hippocampal structures and functions in the present experiment. Specifically, we conducted experiments in the Voal cKO and control mice of ages 5-6 months to determine the persistent effects of Voal deletion.

\section{Impaired Spatial Memory in Voa1 cKO Mice}

We first compared the performances of the Voal cKO and wild type control mice in the Morris water maze task. We used a 9-day protocol consisted of 3 days of visible platform trials, 6 days of hidden platform trials and two-probe tests (Bin et al., 2018; Figure 1A). Data were collected from seven Voal cKO mice and seven control mice (three female and four male mice in each group).

During the visible platform trials, both the Voal cKO and control mice improved significantly in performance over the 3 -day trials, as there were day/trial-dependent reductions in swimming distance $\left(F_{(2,12)}=5.048, p=0.026\right.$ and $F_{(2,12)}=4.813$, $p=0.029)$ and time $\left(F_{(2,12)}=20.899, p<0.001\right.$ and $F_{(2,12)}=4.460$, $p=0.036$ ) in finding the platform (Figures 1B,C). Significant differences between the Voal $\mathrm{cKO}$ and control mice were observed with respect to the distance $\left(F_{(1,12)}=6.558, p=0.025\right)$ and time $\left(F_{(1,12)}=7.028, p=0.021\right)$ traveled to reach the platform (Figures 1B,C).

During the 6-day hidden platform trials, the control mice were improved in both distance $\left(F_{(5,30)}=5.314, p=0.001\right)$ and time $\left(F_{(5,30)}=4.468, p=0.004\right)$ needed to reach the platform; while the Voal cKO mice showed significant improvement in swimming distance $\left(F_{(5,30)}=2.842, p=0.032\right)$ but not in time measures $\left(F_{(5,30)}=2.535, p=0.050\right)$. There were significant differences between the two groups: the Voal cKO mice swam significantly longer distances $\left(F_{(1,12)}=9.464, p=0.01\right)$ and spent more time $\left(F_{(1,12)}=21.261, p=0.001\right)$ than the control mice to reach the platform (Figures 1B,C).

During the probe test 1 and 2, the Voal cKO mice spent significantly less time in the correct quadrant than the controls $(p=0.01$ and $p=0.001$; Figure 1D). Together, these observations suggest impaired spatial learning and memory in the Voal cKO mice. 
A

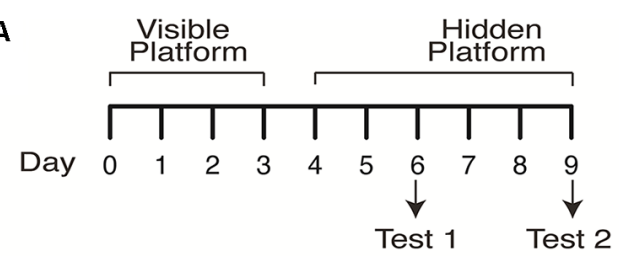

B

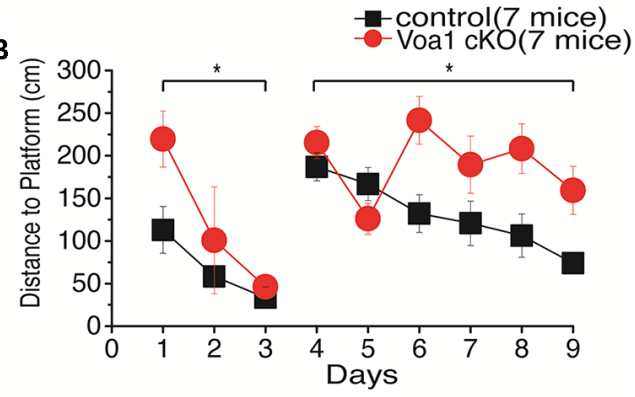

C

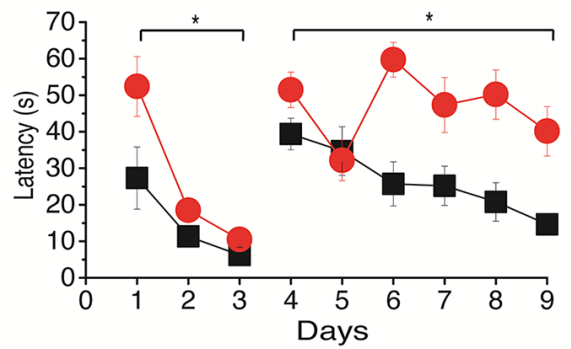

D

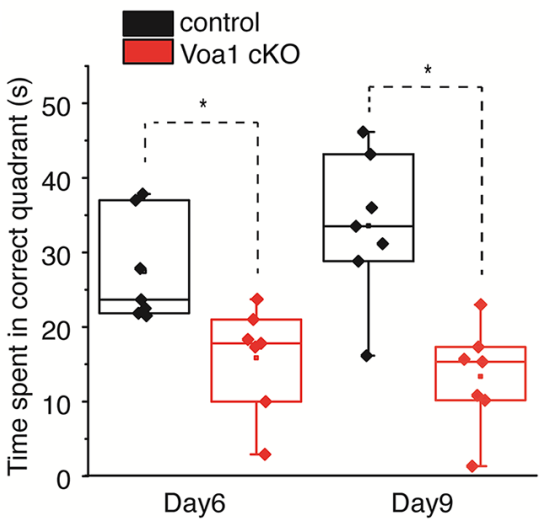

FIGURE 1 | Spatial memory impaired in the Voa1 cKO mice. (A) A maze protocol with 3 days of visible platform trials, 6 days of hidden platform trials and two probe tests was used. Swimming distance and time taken to reach the platform during the visible and hidden platform trials were presented in $\mathbf{( B , C )}$. For the probe tests, the times spent in the correct quadrant where the platform was previously located were measured and presented in (D). There were significant differences between the Voa1 cKO mice and control mice in the distance and time measures during the visible and hidden platform trials (Mixed repeated ANOVA; see "Results" section) as well as in probe tests ( $t$-test). Here and afterwards, group data were presented as means and standard errors of means. Statistical significances $(p \leq 0.05)$ between the Voa1 cKO mice and control mice were denoted by *. Non-significant difference denoted by n.s. Data points in box plots represented the maximal, minimal, $25 \%$ and $75 \%$ measures of individual groups, and horizontal lines and squares in boxes represented median and mean values. Data and responses collected from the Voa1 cKO or control mice were marked by red or black colors.

\section{CA1 Synaptic Field Potentials Were Diminished in Voa1 cKO Mice}

We next examined hippocampal synaptic activities in the Voal cKO mice and focused on the well-characterized Shaffer collateral-CA1 pathway. Extracellular recordings in brain slices were used to assess changes in transmission at a local circuitry level (15-26 slices from four Voal cKO or control mice). Synaptic field potentials were recorded from the CA1 striatum radium which is the apical dendritic layer of the CA1 pyramidal neurons. Paired stimulations $(25 \mathrm{~ms}$ interval) of different intensities were used to examine the overall activity and short-term plasticity of the Shaffer collateral-CA1 pathway. Monitored from dorsal hippocampal areas, CA1 synaptic field potentials of the Voal cKO mice were either unrecognizable or seemingly small amplitudes. The overall amplitudes, evoked by the first and second stimulations were greatly diminished as compared to the controls $\left(F_{(1,39)}=104.553, p<0.001\right.$ and $F_{(1.39)}=142.727$, $p<0.001$; Figures 2A,B).

Despite the great group differences in synaptic field potentials, the amplitudes of presynaptic volleys, which present the overall activity of activated presynaptic fibers of the Schaffer collateral-CA1 pathway, were not significantly different between the Voal $\mathrm{cKO}$ and control mice $\left(F_{(1,39)}=1.226\right.$, $p=0.275$; Figure 2C). These observations suggest that postsynaptic dysfunction may largely account for the diminished CAl synaptic potentials in the Voal cKO mice, which is consistent with the histological assessment described below.

Monitored from ventral hippocampal areas, CA1 field potentials evoked by the first and second stimulations were also decreased in the Voal cKO mice relative to the controls $\left(F_{(1,17)}=10.485, p=0.005\right.$ and $F_{(1,17)}=13.811$, $p=0.002)$; whereas the amplitudes of presynaptic volleys were largely similar between the Voal cKO and controls mice $\left(F_{(1,17)}=0.672, p=0.424\right.$; Figures 2D-F). Overall, the diminished CA1 field potentials in the Voal cKO mice were more pronounced in the dorsal than in ventral hippocampal areas examined (Figures 2B,E). As glutamatergic activities are the predominant component of the CA1 synaptic field potentials, these observations suggest regional alterations of hippocampal CA1 glutamate synapses in the Voal cKO mice. The decreased ventral CA1 field potentials might be largely due to alterations in synaptic transmission as Voa1-containing V-ATPase has been suggested to modulate neurotransmitter (glutamate) release in rat hippocampal neurons (Bodzeta et al., 2017).

\section{Hippocampal CA1 Neurodegeneration in Voa1 cKO Mice}

We also performed general histological experiments to see whether the above functional assessments are associated with evident neuronal degeneration in the Voal cKO mice. Coronal sections $(25 \mu \mathrm{m})$ were stained with cresyl violet for general morphological assessments. In sections encompassing dorsal hippocampal areas (Bregma -1.7 to $-1.94 \mathrm{~mm}$ ), brain hemispheric and hippocampal areas were decreased in the Voal cKO mice $(n=7)$ relative to the control mice $(n=7 ; p=0.006$ or 0.008 ; Figures $3 \mathbf{A}, \mathbf{D})$. In addition, 


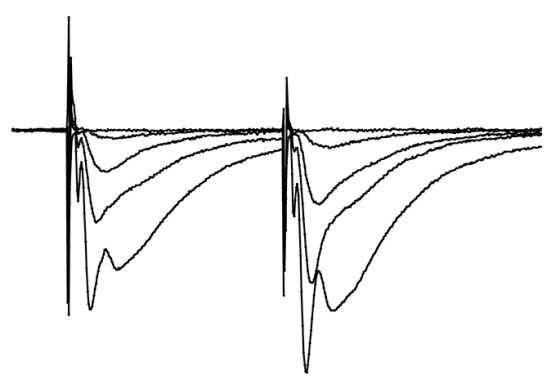

B

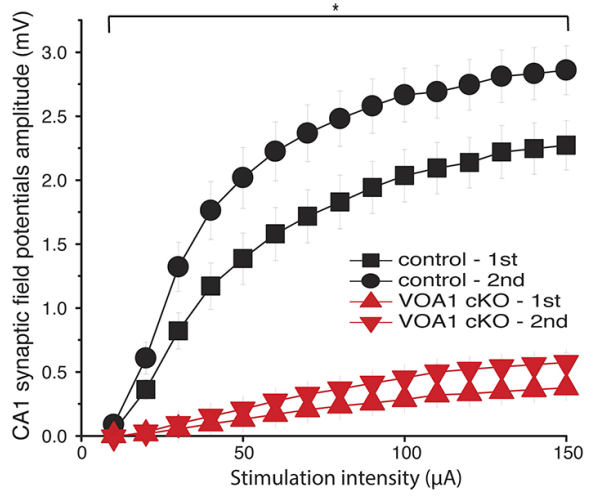

D

Ventral hippocampus

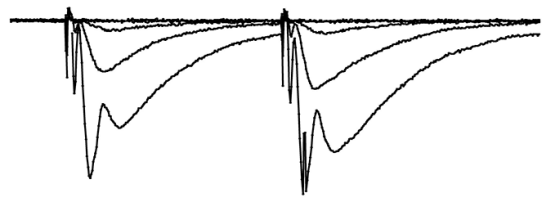

E

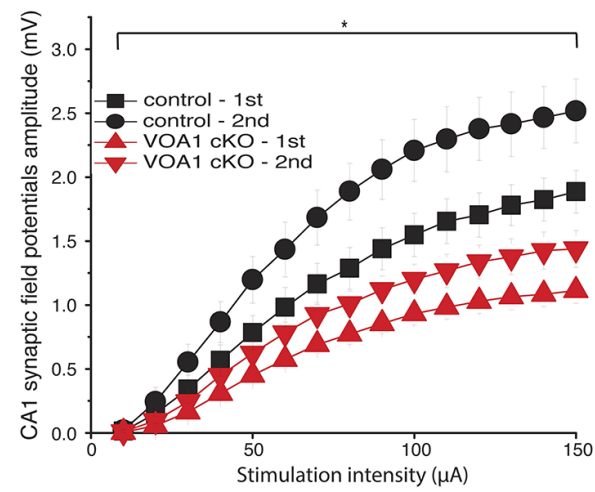

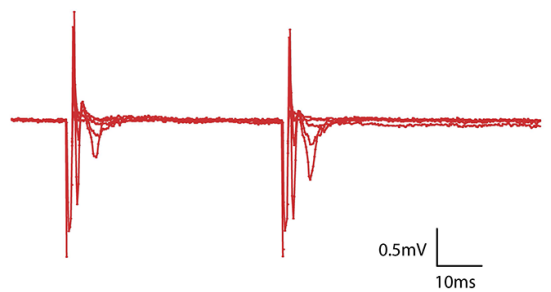

C
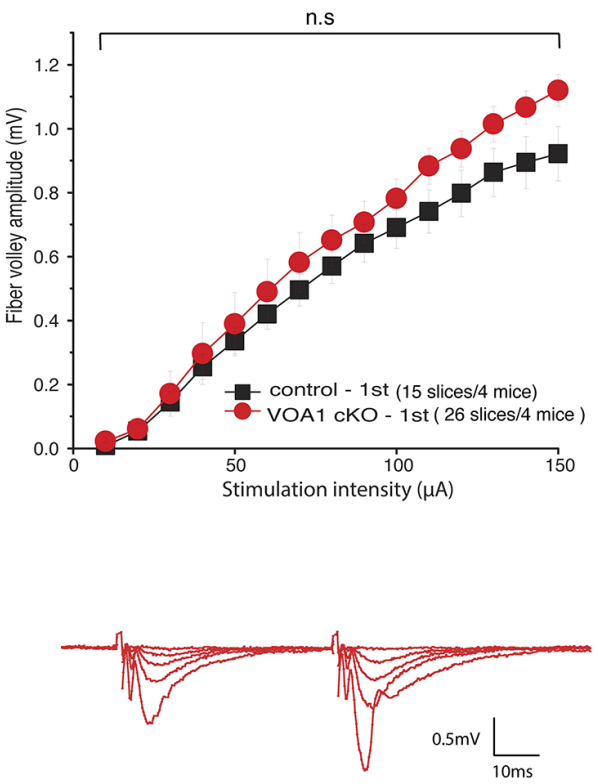

$\mathbf{F}$

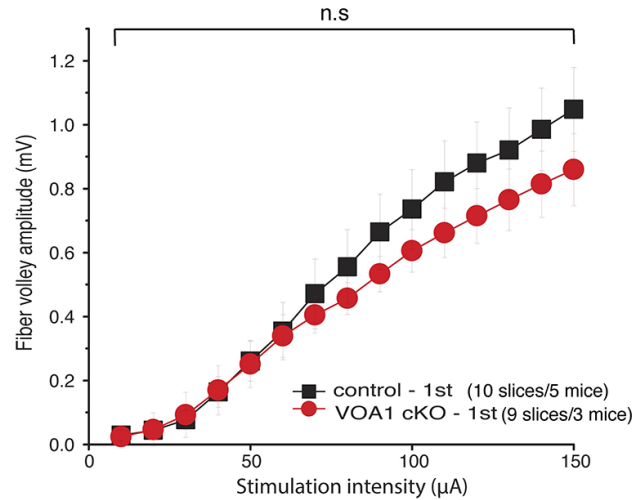

FIGURE 2 | CA1 synaptic field potentials diminished in the Voa1 cKO mice. (A,D) Representative traces were collected from the Voa1 cKO mice and the control mice. Dorsal (A) and ventral (D) CA1 synaptic field potentials were evoked by paired stimulations of the Shaffer collateral pathway at intensities of 10, 20, 30, 50 or $150 \mu \mathrm{A}$, respectively. (B,E) The amplitudes of 1st and 2nd CA1 field potentials were plotted vs. stimulation intensities. (C,F) The amplitudes of presynaptic volleys were plotted vs. stimulation intensities. The synaptic field potentials (B,E), but not the presynaptic volleys $\mathbf{( C , F )}$, were significantly different between the Voa1 cKO

mice and control mice (Mixed repeated ANOVA; see details in "Results" section).

degenerated hippocampal CA1 neurons were evident in all Voal cKO mice examined and featured with vanished or diminished CA1 cell body layer (Figures 3B,C). The overall
CA1 cell density was greatly decreased as compared to the control $(p<0.001$; Figure 3D, left panel). Despite the CA1 degeneration, the CA3 cell body layer appeared to 
A
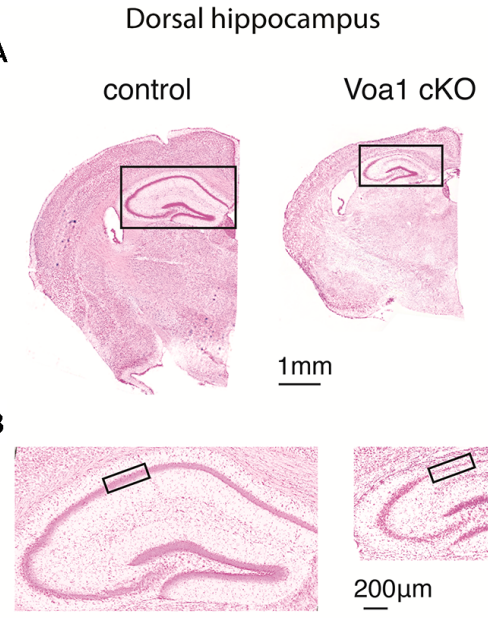

C

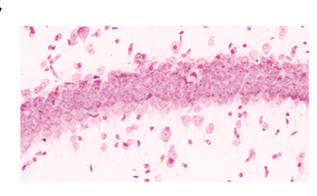

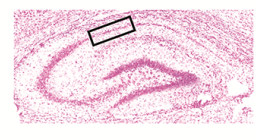

$200 \mu \mathrm{m}$

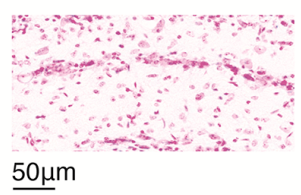

Ventral hippocampus

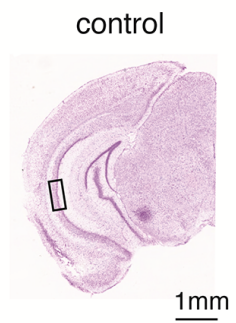

Voa1 cKO

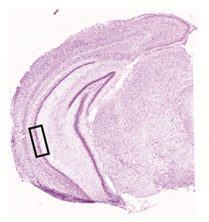

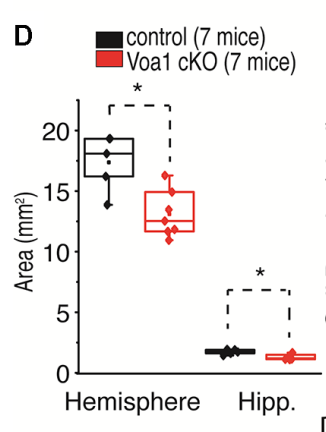
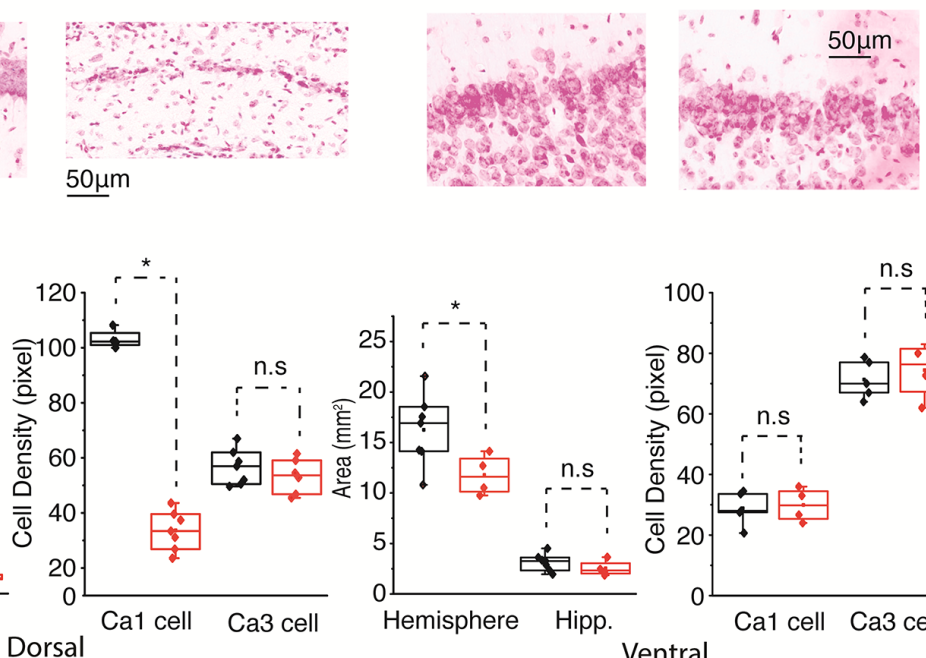

Dorsal
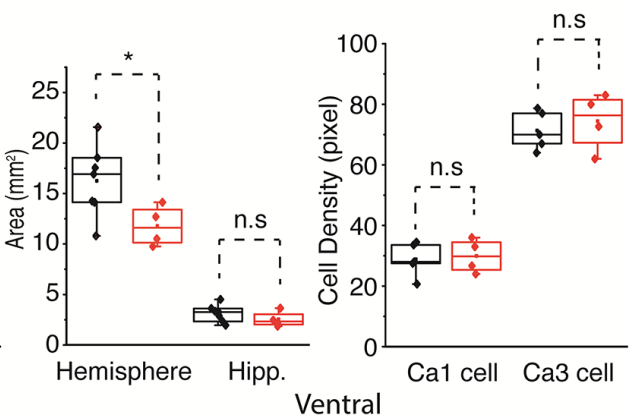

FIGURE 3 | Dorsal hippocampal CA1 neurons degenerated in the Voa1 cKO mice. (A-C) Images were collected from two control mice and two Voa1 cKO mice. Representative coronal sections encompassing dorsal or ventral hippocampal areas were stained with cresyl violet. Squared hippocampal areas in (A) left panels were expanded in (B). Squared CA1 sectors in (B) or in (A) right panel were enlarged in (C) left or right panel. (D)Left and (D) right, hemispheric and hippocampal areas and densities of CA1 and CA3 cell body layers in coronal sections encompassing dorsal and ventral hippocampal areas respectively. Cell densities measures were made from adjacent three sections for each mouse. Data collected from seven Voa1 cKO mice and seven control mice. Note that hemispheric and dorsal hippocampal areas were decreased in the Voa1 $\mathrm{cKO}$ mice relative to the controls (independent $t$-test). Note also that the density of CA1 cell body layer was greatly decreased in the dorsal hippocampus of the Voa1 cKO mice.

be largely preserved in all Voal cKO mice examined. The CA3 cell density measures of the Voa1 cKO mice were not significantly different from the controls $(p=0.373$; Figure 3D, right).

In coronal sections encompassing ventral hippocampal areas (Bregma -3.16 to $-3.4 \mathrm{~mm}$ ), brain hemispheric areas of the Voal cKO mice were moderately reduced relative to the controls ( $p=0.046$ ) but hippocampal areas were not significantly different between the two groups of mice ( $p=0.284$; Figure 3D, right). Ventral hippocampal neurodegeneration was not evident in the Voal cKO mice examined (Figures 3A,C). The ventral CA1 and CA3 cell densities of the Voal cKO mice were not significantly different from the controls $(p=0.784$ and $p=0.573$; Figure 3D, right).

\section{CA3 Hyperexcitable Activities in Voa1 cKO Mice}

The above histological observations motivated us to examine hippocampal CA3 EEG activities from the Voal cKO mice. The CA3 recurrent circuitry has intensive projections along the hippocampal longitudinal axis (Witter, 2007) and such CA3 longitudinal network is critical for general "physiological" and "pathophysiological” hippocampal activities (Buzsáki, 2015) but disrupted in brain slices. We anticipated that EEG recordings from the hippocampal CA3 area may have great propensity of revealing aberrant CA3 network activities in the Voal cKO mice. Because dorsal hippocampal areas were decreased in the Voal cKO mice and it was difficult to target the dorsal hippocampal CA3 in these mice (Figures $\mathbf{3 A}, \mathbf{D}$ ), 
we implanted electrodes in the middle-ventral hippocampal CA3 area to examine potential aberrant EEG activities. EEG recordings from the hippocampal CA3 area revealed spikes in seven Voal cKO mice examined (three female and four male mice; Figures 4 A,C,D). These spikes occurred intermittently during behavioral immobility or sleep, and spike rates were variable among individual mice (up to 93 spikes per $10 \mathrm{~min}$; Figure 4C). Diazepam is a benzodiazepine positive allosteric modulator of the $\mathrm{GABA}_{\mathrm{A}}$ receptors. Previous works from our lab have shown that intraperitoneal injections of diazepam at $1.5 \mathrm{mg} / \mathrm{kg}$ were effective in suppressing hippocampal spikes or seizures in other mouse models (El-Hayek et al., 2011, 2013). We thus examined the effects of diazepam in the Voal mice. A single intraperitoneal injection of diazepam at $1.5 \mathrm{mg} / \mathrm{kg}$ reduced spike rates in five Voal cKO mice tested ( $p=0.026$; Figures $4 \mathrm{~A}, \mathrm{C})$, suggesting that attenuated GABAergic inhibition might partly account for the genesis of aberrant hippocampal spikes in the Voal cKO mice.

In addition to the spikes, two discharge episodes were observed from one Voal cKO mouse (Figure 4B). These discharges lasted 19-22 $\mathrm{s}$ and their occurrence was not associated with evident convulsive behavior. The waveform and behavioral correlate of these discharges were similar to the "nonconvulsive electrographic seizure events" previously observed from post-ischemic mice (Song et al., 2018a). In contrast, neither hippocampal spike nor discharge was observed from five control mice examined (two female and three male mice; Figure 4A). Together these EEG observations suggest that the hippocampal CA3 network activity was altered towards hyperexcitability in the Voal cKO mice.

\section{DISCUSSION}

We examined the Voal cKO mice of ages 5-6 months to determine the persistent effects of Voal deletion. Because $\mathrm{CaMK} \alpha-\mathrm{Cre}$ is expressed in forebrain areas particularly in the hippocampal CA1 (Tsien et al., 1996; Sonner et al., 2005), the focus of our experiments was on structural and functional changes in the hippocampus. The Voal knockout mice exhibited evident hippocampal abnormalities. Specifically, the Voal cKO mice were impaired in spatial learning and memory as they performed poorly relative to the controls in the visible and hidden platform trials and probe tests of the Morris water maze test. In addition, the Voal cKO mice had deficits in hippocampal synaptic functions as the CA1 synaptic potentials recorded in brain slices were greatly diminished. Further, histologically recognized CA1 neurodegeneration was pronounced in the dorsal hippocampus of the Voal cKO mice. The CA1 neurodegeneration was associated with general brain atrophy as overall hemispheric areas were reduced in the Voal cKO mice relative to the controls. Taking together these observations and the notion that the dorsal hippocampus is crucial for spatial learning and memory (Strange et al., 2014), we postulate that the spatial learning and memory impairment observed from the Voal cKO mice may result from the hippocampal CA1 neurodegeneration as well as abnormalities in other brain structures yet to be further characterized.
Such CA1 neurodegeneration may largely result from chronic dis-regulations in cellular $\mathrm{pH}$ and energy metabolisms due to malfunctions of V-ATPase (Forgac, 2007; Cotter et al., 2015; Hayek et al., 2019). While the exact mechanisms and time course by which CA1 neurons degenerate in the Voal cKO mice remain a topic of further investigation, our present observations are in consistent with recent findings that V-ATPase interruption by targeting ATP6AP2 causes neuronal degeneration in other brain regions (Dubos et al., 2015; Fassio et al., 2018; Hirose et al., 2019).

Despite the pronounced CA1 degeneration/dysfunction of the dorsal hippocampus, the hippocampal CA3 neurons appeared to be spared morphologically in the Voal cKO mice. In addition, CA3 hyperexcitable activities were observed from the Voa1 cKO mice as CA3 EEG recordings detected aberrant diazepamsensitive spikes and non-convulsive discharges. These regional differences may mainly result from the discrete Cre expression pattern (Tsien et al., 1996; Sonner et al., 2005) hence the severe Voal deletion in the dorsal hippocampal CA1. The vulnerability of the dorsal hippocampal CA1 neurons, which was previously demonstrated in models of brain hypoxia/ischemia (SchmidtKastner, 2015), may also be a factor. It is presently unknown as to when and how the CA3 hyperexcitability evolves in the Voal cKO mice. One possibility is that the hippocampal CA3 circuitry is altered towards hyperexcitability to compensate the degenerated/dysfunctional CA1 circuitry. Previous studies have demonstrated the hippocampal CAl degeneration and CA3 hyperexcitability in a rat model of transient global brain ischemia (Wu et al., 2005; Epsztein et al., 2006). When examined a few months after initial ischemic epidotes, rats presented a complete loss of dorsal hippocampal CA1 neurons and robust CA3 EEG spikes or hyperexcitable responses. Together with our present observations and these previous studies raise an intriguing possibility as to whether the CA3 hyperexcitability is a common chronic outcome in rodent models with CA1 neuronal injury/degeneration. Another possibility is that the CA3 hyperexcitability starts prior to or concurs with the CA1 degeneration process hence playing a critical role in the CA1 neurodegeneration through excitotoxicity mechanisms (Lai et al., 2014). As Cre recombinase expression in hippocampus stabilizes after postnatal 19-20 days (Tsien et al., 1996), future experiments that examine the Voal cKO mice at different time points during postnatal development may help determine the temporal relation of CA1 degeneration and CA3 hyperexcitability.

Our present experiments have limitations and complications. In particular, the expression of Cre recombinase gene under CaMKII $\alpha$ promoter caused severe degeneration of dorsal hippocampal CA1 neurons in the Voal cKO mice (see above). While the precise timing of CA1 degeneration was unknown, it was difficult for us to investigate whether Voal protein is decreased while Voal cKO CA1 neurons were still alive using brain sections encompassing dorsal hippocampal areas. Therefore, we decided to use cultured hippocampal neurons as an alternative approach (see "Materials and Methods" section). We infected floxed neurons with lentiviral particles that co-expressed GFP and blasticidin S resistance gene (control) or GFP and Cre recombinase gene (KO) on (DIV 1). As KO neurons started to 
A

control

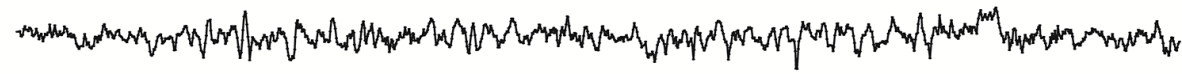

Voa1 $\mathrm{KO}$

4hom

diazepam

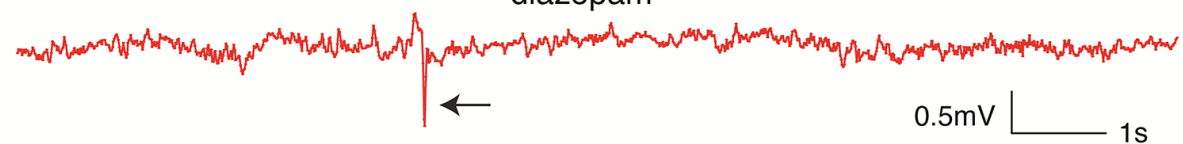

B

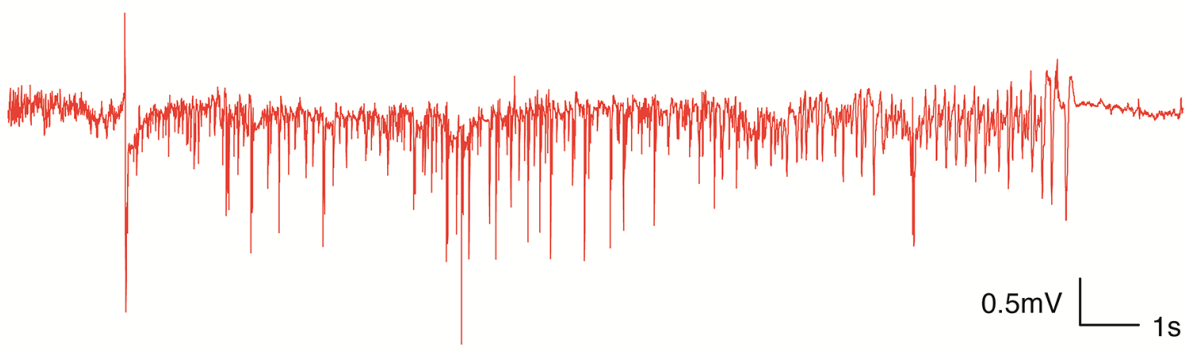

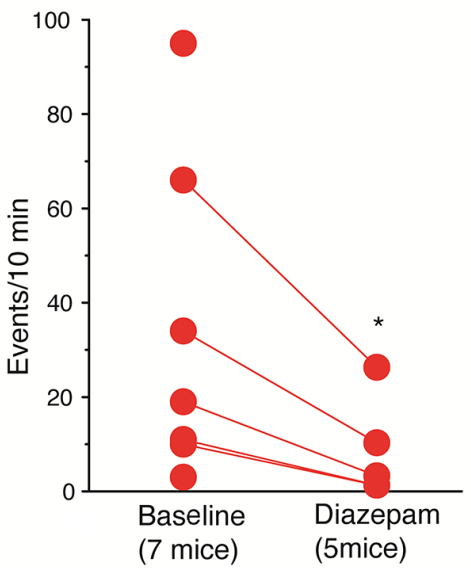

D

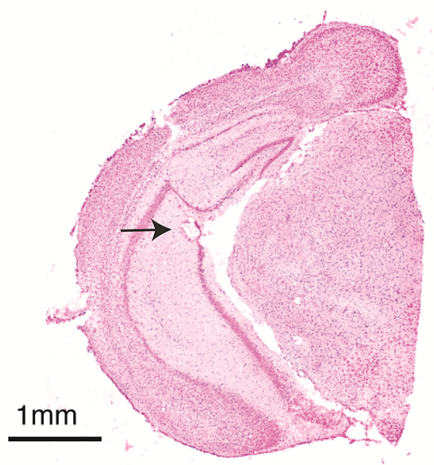

FIGURE 4 | Aberrant CA3 electroencephalographic (EEG) activities observed from the Voa1 cKO mice. (A) Representative CA3 EEG traces collected from a control mouse and a Voa1 CKO mouse. Examples of spikes were denoted by filled arrows. Note multiple spikes and a single spike recorded from the Voa1 cKO mouse before and about $2 \mathrm{~h}$ following diazepam injection. (B) An episode of CA3 discharge collected from another Voa1 cKO mouse. (C) Spike rates (events/10 min) measured from seven Voa1 cKO mice. Data collected from five of the seven mice following saline (baseline) and diazepam injections were linked by solid lines. Note a significant decrease in spike rate following the diazepam injections (paired $t$-test). (D) A representative cresyl violet-stained section showing the track and putative tip location (filled arrow) of an implanted hippocampal electrode.

show signs of deterioration on DIV 13, we harvested control and KO neurons on DIV 13 for Western blot analysis. These experiments demonstrated a clear reduction of Voal in $\mathrm{KO}$ neurons (Supplementary Figure S1B), suggesting that reduction of Voal may take place prior to neurodegeneration. In addition, we used C57BL/6 mice with CaMKII $\alpha$-Cre (Tsien et al., 1996) to generate the Voal cKO model. Naïve C57 black mice are known to perform well in the Morris water maze test (Wolff et al., 2002; Patil et al., 2009; Kapadia et al., 2016), but experimental factors including the influences by sex and stress responses can substantially affect animal performance in the Morris water maze test (Kapadia et al., 2016). As we used mixed female and male mice in the present experiments, the influences by sex and stress responses on spatial memory outcomes as well as on CA3 EEG signals remain to be evaluated. Moreover, as the Cre recombinase under CaMKII $\alpha$ promoter is expected to delete Voa1 in forebrain areas in addition to the dorsal hippocampal CA1, structural and/or functional abnormalities in these forebrain areas might also affect the performance of the Voal cKO mice in the Morris water maze test and cause other behavioral abnormalities. Future experiments conducting hippocampus-dependent and independent tasks are needed to fully characterize functional 
deficits in the Voal cKO mice. The lack of investigation on molecular and cellular mechanisms of the CA1 degeneration and CA3 hyperexcitability in the Voa $1 \mathrm{cKO}$ mice is a major weakness of our present study.

Despite these limitations and weaknesses, the Voal knockout caused severe neurodegeneration in the targeted dorsal hippocampal CA1, suggesting that Voal related activities are essential for the survival of mammalian central neurons. While severe neurodegeneration makes it impossible for us to examine the precise role of this protein in the process of neurotransmitter release (Saw et al., 2011; Bodzeta et al., 2017), the Voal cKO mice may complement other models (Dubos et al., 2015; Fassio et al., 2018; Hirose et al., 2019) for further investigation of V-ATPase dysfunction related neuronal degeneration.

\section{DATA AVAILABILITY STATEMENT}

All data generated or analyzed during this study are included in this published article and are available from the corresponding author on reasonable request.

\section{ETHICS STATEMENT}

The animal study was reviewed and approved by the animal care committee of the University Health Network.

\section{AUTHOR CONTRIBUTIONS}

All authors were involved in experimental design, data discussion and interpretation. Specifically, KM, N-RB, SSh and $\mathrm{HH}$ conducted experiments and data analysis. YW and G-H-SW generated and characterized Voal antibody. N-RB, SSh, SSu, $\mathrm{PM}$ and LZ were responsible for manuscript writing and

\section{REFERENCES}

Bagh, M. B., Peng, S., Chandra, G., Zhang, Z., Singh, S. P., Pattabiraman, N., et al. (2017). Misrouting of v-ATPase subunit V0a1 dysregulates lysosomal acidification in a neurodegenerative lysosomal storage disease model. Nat. Commun. 8:14612. doi: 10.1038/ncomms14612

Bin, N. R., Ma, K., Harada, H., Tien, C. W., Bergin, F., Sugita, K., et al. (2018). Crucial role of postsynaptic syntaxin 4 in mediating basal neurotransmission and synaptic plasticity in hippocampal CA1 neurons. Cell Rep. 23, 2955-2966. doi: 10.1016/j.celrep.2018.05.026

Bin, N. R., Song, H., Wu, C., Lau, M., Sugita, S., Eubanks, J. H., et al. (2017). Continuous monitoring via tethered electroencephalography of spontaneous recurrent seizures in mice. Front. Behav. Neurosci. 11:172. doi: 10.3389/fnbeh. 2017.00172

Bodzeta, A., Kahms, M., and Klingauf, J. (2017). The presynaptic v-ATPase reversibly disassembles and thereby modulates exocytosis but is not part of the fusion machinery. Cell Rep. 20, 1348-1359. doi: 10.1016/j.celrep.2017.07.040

Buzsáki, G. (2015). Hippocampal sharp wave-ripple: a cognitive biomarker for episodic memory and planning. Hippocampus 25, 1073-1188. doi: 10.1002/hipo.22488

Chanaday, N. L., and Kavalali, E. T. (2018). Optical detection of three modes of endocytosis at hippocampal synapses. Elife 7:e36097. doi: 10.7554/eLife. 36097

Colacurcio, D. J., and Nixon, R. A. (2016). Disorders of lysosomal acidification-The emerging role of v-ATPase in aging and neurodegenerative disease. Ageing Res. Rev. 32, 75-88. doi: 10.1016/j.arr.2016.05.004 figure assembling. The submitted manuscript was reviewed and approved by all authors.

\section{FUNDING}

This work was supported by research grants from Canadian Institute of Health Research (MOP-93665, 130573), Natural Science and Engineering Research Council of Canada (RGPIN 217479-10 and RGPIN-2015-06438) and Ontario Brain Institute (Eplink program).

\section{SUPPLEMENTARY MATERIAL}

The Supplementary Material for this article can be found online at: https://www.frontiersin.org/articles/10.3389/fncel.2019.004 84/full\#supplementary-material.

FIGURE S1 | Generation of Voa1 conditional knockout mice and knockout neurons. (A) Top panel, a schematic diagram of the Voa1 floxed allele (labeled as flox) in comparison with wild type (WT) allele; lower panels, genotyping PCR to detect WT vs. floxed allele (left) and CaMKIl $\alpha$-Cre recombinase gene (right). (B) Western blot analysis shows a clear reduction in Voa1 protein in cultured floxed neurons on days in vitro 13 (DIV 13) after application of Cre recombinase to the neurons on DIV 1. The level of ubiquitously expressed VCP protein and neuron-specific cytosolic Munc18-1 protein is unchanged.

FIGURE S2 | Immunofluorescence images of Voa1 hippocampal expression in wild type mice. Images were obtained from wild type mice of 6 months-old. Top-left, a brain section stained with the goat anti-chick antibody alone. Hippocampal subfields and enlarged CA1 and CA3 areas were presented as indicated. Bottom-left, another brain section stained with the chicken monoclonal Voa1 antibody lgY and then with the goat anti-chick antibody. Images were similarly presented as in the top panel. Top-right and Bottom-right, images from same sections showing DAPI staining (blue). Note strong Voa1 signals in hippocampal subfields and enlarged CA1 and CA3 areas in images of the Bottom-left panel.

Cotter, K., Stransky, L., McGuire, C., and Forgac, M. (2015). Recent insights into the structure, regulation, and function of the V-ATPases. Trends Biochem. Sci. 40, 611-622. doi: 10.1016/j.tibs.2015.08.005

Dickinson, M. E., Flenniken, A. M., Ji, X., Teboul, L., Wong, M. D., White, J. K., et al. (2016). High-throughput discovery of novel developmental phenotypes. Nature 537, 508-514. doi: 10.1038/nature19356

Dubos, A., Castells-Nobau, A., Meziane, H., Oortveld, M. A., Houbaert, X., Iacono, G., et al. (2015). Conditional depletion of intellectual disability and Parkinsonism candidate gene ATP6AP2 in fly and mouse induces cognitive impairment and neurodegeneration. Hum. Mol. Genet. 24, 6736-6755. doi: $10.1093 / \mathrm{hmg} / \mathrm{ddv} 380$

El-Hayek, Y. H., Wu, C. P., Chen, R., Al-Sharif, A. R., Huang, S., Patel, N., et al. (2011). Acute postischemic seizures are associated with increased mortality and brain damage in adult mice. Cereb. Cortex 21, 2863-2875. doi: 10.1093/cercor/bhr080

El-Hayek, Y. H., Wu, C. P., Ye, H., Wang, J., Carlen, P. L., and Zhang, L. (2013). Hippocampal excitability is increased in aged mice. Exp. Neurol. 247, 710-719. doi: 10.1016/j.expneurol.2013.03.012

Epsztein, J., Milh, M., Bihi, R. I., Jorquera, I., Ben-Ari, Y., Represa, A., et al. (2006). Ongoing epileptiform activity in the post-ischemic hippocampus is associated with a permanent shift of the excitatory-inhibitory synaptic balance in CA3 pyramidal neurons. J. Neurosci. 26, 7082-7092. doi: 10.1523/JNEUROSCI. 1666-06.2006

Fassio, A., Esposito, A., Kato, M., Saitsu, H., Mei, D., Marini, C., et al. (2018). De novo mutations of the ATP6V1A gene cause developmental encephalopathy with epilepsy. Brain 141, 1703-1718. doi: 10.1093/brain/awy092 
Forgac, M. (2007). Vacuolar ATPases: rotary proton pumps in physiology and pathophysiology. Nat. Rev. Mol. Cell Biol. 8, 917-929. doi: 10.1038/ $\operatorname{nrm} 2272$

Franklin, K. B. J., and Paxinos, G. (1997). The Mouse Brain in Stereotaxic Coordinates. San Diego, CA: Academic Press.

Hayek, S. R., Rane, H. S., and Parra, K. J. (2019). Reciprocal regulation of V-ATPase and glycolytic pathway elements in health and disease. Front. Physiol. 10:127. doi: 10.3389/fphys.2019.00127

Hiesinger, P. R., Fayyazuddin, A., Mehta, S. Q., Rosenmund, T., Schulze, K. L., Zhai, R. G., et al. (2005). The v-ATPase V0 subunit a1 is required for a late step in synaptic vesicle exocytosis in Drosophila. Cell 121, 607-620. doi: 10.1016/j. cell.2005.03.012

Hirose, T., Cabrera-Socorro, A., Chitayat, D., Lemonnier, T., Féraud, O., Cifuentes-Diaz, C., et al. (2019). ATP6AP2 variant impairs CNS development and neuronal survival to cause fulminant neurodegeneration. J. Clin. Invest. 130, 2145-2162. doi: 10.1172/jci79990

Kapadia, M., Xu, J., and Sakic, B. (2016). The water maze paradigm in experimental studies of chronic cognitive disorders: theory, protocols, analysis, and inference. Neurosci. Biobehav. Rev. 68, 195-217. doi: 10.1016/j.neubiorev. 2016.05.016

Kavalali, E. T., Klingauf, J., and Tsien, R. W. (1999). Activity-dependent regulation of synaptic clustering in a hippocampal culture system. Proc. Natl. Acad. Sci. U S A 96, 12893-12900. doi: 10.1073/pnas.96.22.12893

Lai, T. W., Zhang, S., and Wang, Y. T. (2014). Excitotoxicity and stroke: identifying novel targets for neuroprotection. Prog. Neurobiol. 115, 157-188. doi: 10.1016/j. pneurobio.2013.11.006

Lee, J. H., McBrayer, M. K., Wolfe, D. M., Haslett, L. J., Kumar, A., Sato, Y., et al. (2015). Presenilin 1 maintains lysosomal $\mathrm{Ca}^{2+}$ homeostasis via TRPML1 by regulating vATPase-mediated lysosome acidification. Cell Rep. 12, 1430-1444. doi: 10.1016/j.celrep.2015.07.050

Moriyama, Y., and Futai, M. (1990). $\mathrm{H}^{+}$-ATPase, a primary pump for accumulation of neurotransmitters, is a major constituent of brain synaptic vesicles. Biochem. Biophys. Res. Commun. 173, 443-448. doi: 10.1016/s0006$291 x(05) 81078-2$

Nishi, T., and Forgac, M. (2000). Molecular cloning and expression of three isoforms of the $100-\mathrm{kDa}$ a subunit of the mouse vacuolar proton-translocating ATPase. J. Biol. Chem. 275, 6824-6830. doi: 10.1074/jbc.275.10.6824

Patil, S. S., Sunyer, B., Höger, H., and Lubec, G. (2009). Evaluation of spatial memory of C57BL/6J and CD1 mice in the Barnes maze, the multiple T-maze and in the Morris water maze. Behav. Brain Res. 198, 58-68. doi: 10.1016/j.bbr. 2008.10.029

Peri, F., and Nüsslein-Volhard, C. (2008). Live imaging of neuronal degradation by microglia reveals a role for v0-ATPase a1 in phagosomal fusion in vivo. Cell 133, 916-927. doi: 10.1016/j.cell.2008.04.037

Perin, M. S., Fried, V. A., Stone, D. K., Xie, X. S., and Südhof, T. C. (1991). Structure of the $116-\mathrm{kDa}$ polypeptide of the clathrin-coated vesicle/synaptic vesicle proton pump. J. Biol. Chem. 266, 3877-3881.

Peters, C., Bayer, M., Bühler, S., Andersen, J. S., Mann, M., and Mayer, A. (2001). Trans-complex formation by proteolipid channels in the terminal phase of membrane fusion. Nature 409, 581-588. doi: 10.1038/35054500

Saw, N. M., Kang, S. Y., Parsaud, L., Han, G. A., Jiang, T., Grzegorczyk, K., et al. (2011). Vacuolar $\mathrm{H}^{+}$-ATPase subunits Voa1 and Voa2 cooperatively regulate secretory vesicle acidification, transmitter uptake, and storage. Mol. Biol. Cell 22, 3394-3409. doi: 10.1091/mbc.e11-02-0155
Schmidt-Kastner, R. (2015). Genomic approach to selective vulnerability of the hippocampus in brain ischemia-hypoxia. Neuroscience 309, 259-279. doi: 10.1016/j.neuroscience.2015.08.034

Song, H., Mylvaganam, S. M., Wang, J., Mylvaganam, S. M. K., Wu, C., Carlen, P. L., et al. (2018a). Contributions of the hippocampal CA3 circuitry to acute seizures and hyperexcitability responses in mouse models of brain ischemia. Front. Cell. Neurosci. 12:278. doi: 10.3389/fncel.2018.00278

Song, H., Tufa, U., Chow, J., Sivanenthiran, N., Cheng, C., Lim, S., et al. (2018b). Effects of antiepileptic drugs on spontaneous recurrent seizures in a novel model of extended hippocampal kindling in mice. Front. Pharmacol. 9:451. doi: 10.3389/fphar.2018.00451

Sonner, J. M., Cascio, M., Xing, Y., Fanselow, M. S., Kralic, J. E., Morrow, A. L., et al. (2005). $\alpha 1$ subunit-containing GABA type A receptors in forebrain contribute to the effect of inhaled anesthetics on conditioned fear. Mol. Pharmacol. 68, 61-68. doi: 10.1124/mol.104.009936

Strange, B. A., Witter, M. P., Lein, E. S., and Moser, E. I. (2014). Functional organization of the hippocampal longitudinal axis. Nat. Rev. Neurosci. 15, 655-669. doi: 10.1038/nrn3785

Sun-Wada, G. H., Tabata, H., Kuhara, M., Kitahara, I., Takashima, Y., and Wada, Y. (2011). Generation of chicken monoclonal antibodies against the a1, a2, and a3 subunit isoforms of vacuolar-type proton ATPase. Hybridoma 30, 199-203. doi: 10.1089/hyb.2010.0087

Toyomura, T., Oka, T., Yamaguchi, C., Wada, Y., and Futai, M. (2000). Three subunit a isoforms of mouse vacuolar $\mathrm{H}^{+}$-ATPase. J. Biol. Chem. 275, 8760-8765. doi: 10.1074/jbc.275.12.8760

Tsien, J. Z., Chen, D. F., Gerber, D., Tom, C., Mercer, E. H., Anderson, D. J., et al. (1996). Subregion- and cell type-restricted gene knockout in mouse brain. Cell 87, 1317-1326. doi: 10.1016/s0092-8674(00)81826-7

Wang, D., Epstein, D., Khalaf, O., Srinivasan, S., Williamson, W. R., Fayyazuddin, A., et al. (2014). $\mathrm{Ca}^{2+}$-Calmodulin regulates SNARE assembly and spontaneous neurotransmitter release via v-ATPase subunit V0a1. J. Cell Biol. 205, 21-31. doi: 10.1083/jcb.201312109

Witter, M. P. (2007). Intrinsic and extrinsic wiring of CA3: indications for connectional heterogeneity. Learn. Mem. 14, 705-713. doi: 10.1101/lm.725207

Wolff, M., Savova, M., Malleret, G., Segu, L., and Buhot, M. C. (2002). Differential learning abilities of $129 \mathrm{~T} 2 / \mathrm{Sv}$ and $\mathrm{C} 57 \mathrm{BL} / 6 \mathrm{~J}$ mice as assessed in three water maze protocols. Behav. Brain Res. 136, 463-474. doi: 10.1016/s01664328(02)00192-4

Wu, C. P., Cheung, G., Rakhshani, N., Parvardeh, S., Asl, M. N., Huang, H. L., et al. (2005). Ca3 neuronal activities of dorsal and ventral hippocampus are differentially altered in rats after prolonged post-ischemic survival. Neuroscience 130, 527-539. doi: 10.1016/j.neuroscience.2004.09.041

Conflict of Interest: The authors declare that the research was conducted in the absence of any commercial or financial relationships that could be construed as a potential conflict of interest.

Copyright (C) 2019 Ma, Bin, Shi, Harada, Wada, Wada, Monnier, Sugita and Zhang. This is an open-access article distributed under the terms of the Creative Commons Attribution License (CC BY). The use, distribution or reproduction in other forums is permitted, provided the original author(s) and the copyright owner(s) are credited and that the original publication in this journal is cited, in accordance with accepted academic practice. No use, distribution or reproduction is permitted which does not comply with these terms. 